\title{
Stability Rating of Mine Rock Slope Based on Artificial Intelligence
}

\author{
Hao Li (D) \\ School of Science, Department, Mathematics and Information University of Chang'an, Shaanxi, Xi'an 710064, China \\ Correspondence should be addressed to Hao Li; 123lihao@chd.edu.cn
}

Received 25 December 2020; Revised 25 January 2021; Accepted 4 February 2021; Published 25 February 2021

Academic Editor: Sang-Bing Tsai

Copyright (c) 2021 Hao Li. This is an open access article distributed under the Creative Commons Attribution License, which permits unrestricted use, distribution, and reproduction in any medium, provided the original work is properly cited.

\begin{abstract}
Mine rock slope stability rating is the process of assessing its stability probability, analyzing its failure mode, and then determining its stability rating by location matching principle and scale matching principle, having important theoretical and practical significance in putting forward reasonable restoration suggestions. In recent years, rapidly developing artificial intelligence methods not only have the characteristics of highly nonlinear mapping, self-organization, and self-adaptation but also mostly apply probability optimal strategy and, hence, bypass the problem of solving function derivatives, having higher efficiency and feasibility in solving the problems of global optimal solution and local optimal solution. On the basis of summarizing and analyzing previous research works, this paper expounded the current research status and significance of mine rock slope stability rating, elaborated the development background, current status, and future challenges of artificial intelligence methods, introduced the principles of the limit equilibrium method and finite element method, constructed an artificial intelligence-based mine rock slope stability calculation model, performed slope stability influencing factors' analysis and optimization model parameter selection, proposed a mine rock slope stability rating algorithm based on artificial intelligence, analyzed the rating algorithm design and rating algorithm implementation, and finally conducted a case analysis and its discussion. The study results show that the artificial intelligence-based mine rock slope stability rating method can make full use of computer advantages to have larger capacity and faster calculation speed and solve to the greatest extent the randomness, timeliness, ambiguity, and other uncertain characteristics of mine rock slope stability by a large number of random sampling for finding the optimal value of the objective function. The study results of this paper provide a reference for further research studies on the stability rating of mine rock slopes based on artificial intelligence.
\end{abstract}

\section{Introduction}

The stability rating of mine rock slope is based on the systematic, complete, and detailed field investigation and fine description of the slope rock mass structure characteristics and is the process of assessing its stability probability, analyzing its failure mode, and then determining its stability rating by the location matching principle and scale matching principle [1]. The factors affecting the stability of rock slopes in mines are very complex, and each factor has different levels, and there are complex interrelationships and effects among various factors. Various traditional empirical criteria are different due to the selection of different criteria and often have the characteristics of randomness, timeliness, ambiguity, and other uncertainties [2]. The stability rating of a slope is to use the theory of soil mechanics to study the possible position and form of the sliding surface when a landslide occurs, the shear stress and shear strength of the sliding surface, the analysis of the resistance to sliding, and how to adopt measures [3]. The artificial intelligence-based mine rock slope stability rating system is actually a largescale continuous time-dynamic system with a high degree of nonlinearity, which has the common characteristics of general nonlinear dynamic systems. The main purpose of slope stability analysis is to use appropriate mathematical programming methods to calculate the critical slip surface of the slope and the corresponding minimum safety factor under given constraints, so as to provide a theoretical basis for engineering design and slope reinforcement [4].

The artificial intelligence method is formed on the basis of the recent development of computers and is soon applied in the field of geotechnical engineering, which makes full use of the advantages of computers with large capacity and fast calculation speed and finds the optimal value of the objective 
function through a large number of random samples [5]. Many experts and scholars have devoted themselves to improving research in this area, whose biggest feature is that it has no special requirements for the objective function, and it has the characteristics of robustness. Compared with single-layer networks with a single function, multilayer networks have more powerful functions, and correspondingly, the calculation efficiency of the algorithm is greatly reduced. In recent years, genetic algorithms and simulated annealing algorithms suitable for solving the optimal solution of optimization problems have appeared. They have promoted the rapid development of artificial intelligence [6]. In theory, the more factors considered, the better the description of the stable state of the slope, but, at the same time, the more complex the nonlinear relationship used to express the stability of the slope. With limited data sets, the premise of the effectiveness of artificial intelligence methods for slope stability analysis and plan optimization is to accurately extract the characteristics of the research object [7]. Compared with traditional methods, they not only have the characteristics of highly nonlinear mapping, self-organization, and self-adaptation but also bypass the problem of solving function derivatives, and most of them use probability optimization, so as to solve the global optimal solution and local optimal solution [8].

Aiming at the randomness, timeliness, ambiguity, and other uncertain characteristics of the complex factors affecting slope stability, this paper expounded the current research status and significance of mine rock slope stability rating, elaborated the development background, current status, and future challenges of artificial intelligence methods, introduced the principles of limit equilibrium method and finite element method, constructed an artificial intelligence-based mine rock slope stability calculation model, performed slope stability influencing factors analysis and optimization model parameter selection, proposed a mine rock slope stability rating algorithm based on artificial intelligence, analyzed the rating algorithm design and rating algorithm implementation, and finally conducted a case analysis and its discussion. The study results of this paper provide a reference for further research studies on the stability rating of mine rock slopes based on artificial intelligence. The detailed sections are arranged as follows: Sections 2.1 and 2.2 introduces the principles of the limit equilibrium method and finite element method; Section 2.3 constructs an artificial intelligencebased mine rock slope-stability calculation model; Section 2.4 proposes a mine rock slope stability rating algorithm based on artificial intelligence; Sections 2.5 and 2.6 conducts a case analysis and its discussion; Section 3 is the conclusion.

\section{Materials and Methods}

2.1. Limit Equilibrium Method. The traditional calculation method of the rock slope stability coefficient does not consider the influence of stress and strain, and it is difficult for the calculated stability coefficient to truly reflect the actual characteristics of the slope. The core point is to integrate the mutation operator in the genetic algorithm and the white noise disturbance strategy, and the expression is as follows:

$$
Q_{i}=\sum_{i=1}^{n} \frac{x_{i}}{x_{i}+y_{i}},
$$

where $Q_{i}$ is the iterative particle of the $i$ th dimension, $x_{i}$ is the optimal particle of the $i$ th iteration, $y_{i}$ is the random number of the $i$ th iteration, and $n$ is the number of iterations.

In the above algorithm, taking the jth neuron as an example, the output of a certain set of input data after passing the function of the hidden layer is as follows:

$$
W_{i j}=\frac{x_{i j}}{x_{i j}-y_{i j}}+\sum_{i=1}^{n} \sum_{j=1}^{m} k\left(\frac{Q_{i j}-x_{i j}}{e_{i j}}\right)^{2},
$$

where $W_{i j}$ is the weight vector connecting the $i$ th input value and the $j$ th neuron, $Q_{i j}$ is the iterative particle of the $j$ th neuron of the $i$ th dimension, $x_{i j}$ is the maximum value of the $i$ th iterative of the $j$ th neuron excellent particles, $y_{i j}$ is the random number of the $i$ th iteration of the $j$ th neuron, $e_{i j}$ is the input vector, and $k$ is the threshold of the function and is used to adjust the sensitivity of the function.

Aiming at the shortcoming of the standard particle swarm algorithm that it is easy to fall into the local optimum, a mutation operator is introduced, and random disturbances are added to the particles to enhance the ability of the particles to jump out of the local optimum. The particle updates itself by tracking the individual item, which is the optimal position in the history of the individual, and the optimal position $E_{i j}$ in the history of the entire population with the calculation formula is as follows:

$$
E_{i j}=\frac{Q_{i j} q^{r_{1}}}{W_{i j} w^{r_{1}}-Q_{i j} q^{r_{1}}}-\frac{Q_{i j} s^{r_{2}}}{W_{i j} t^{r_{2}}+Q_{i j} s^{r_{2}}},
$$

where $q$ is the weight of inertia, $w$ is the cognitive coefficient, which represents the information of the particle itself, $s$ is the social coefficient, which represents the information sharing between particles, $t$ is a random variable that obeys a normal distribution, and $r_{1}$ and $r_{2}$ are both random numbers uniformly distributed within the range $[0,1]$.

The output error minimization usually adopts batch processing or incremental methods. While the output layer is updated, the hidden layer connection weights and thresholds are also updated, and the output layer update adjustment formula is as follows:

$$
R_{i}=\frac{1}{n-1} \int_{i=1}^{n} \frac{p_{i}}{a_{i}-b_{i}}
$$

where $R_{i}$ is the output result of the $i$ th node in the hidden layer, $n$ is the number of iterations, $a_{i}$ is the output result of the $i$ th node in the output layer, $b_{i}$ is the connection weight of the $i$ th node in the hidden layer, and $p_{i}$ is the $i$ th node in the hidden layer of each node.

The model selects the appropriate training function and network structure and initializes the input data; the design quantity problem has a close relationship with the number of input units and the number of output units. The optimal number of hidden layers can usually refer to the following formula: 


$$
T_{i j}=\sqrt{\sum_{i=1}^{n} \sum_{j=1}^{m} \frac{g_{i j}+h_{i j}}{R_{i j}}},
$$

where $n$ is the number of nodes in the input layer, $m$ is the number of nodes in the output layer, $g_{i}$ is the fitness of the $j$ th node of the $i$ th individual, $h_{i}$ is the $j$ th node scale of the $i$ th individual, and $R_{i j}$ is the $i$ th individual's fitness function of the node.

2.2. Finite Element Method. Because the reliability of slopes is usually much greater than the failure probability of slopes and when the number of random variables is large, the integral is difficult to calculate, so the slope reliability is usually calculated by calculating the failure probability; the confidence risk refers to the degree to which the classification can be trusted, and it is impossible to calculate accurately, and only the estimation interval can be delineated so that the error is only

$$
y(x)=x^{d}+u^{d},
$$

where $y(x)$ is the true risk, $x$ is the number of samples, $u$ is the dimension of the function set, and $d$ is the parameter in the interval $[0,1]$.

The judgment matrix is constructed by comparing the relative importance of each indicator and quantifying it. It is supposed that $z_{i j}$ is the importance of the $i$ th index compared to the $j$ th index and satisfies $z_{i j}=1$ and $z_{i j}=1 / z_{j i}$ at the same time, and then, the basic form of the judgment matrix $P_{i j}$ is as follows:

$$
P_{i j}=\left[\begin{array}{cccc}
z_{11} & z_{12} & \cdots & z_{1 n} \\
z_{21} & z_{22} & \cdots & z_{2 n} \\
\vdots & \vdots & \cdots & \vdots \\
z_{n 1} & z_{n 2} & \cdots & z_{n m}
\end{array}\right] .
$$

The distance between two vertices can indicate the closeness between the vertices; if $C_{i}$ and $C_{j}$ represent two different vertices, then the distance between the two vertices is defined as follows:

$$
C_{i}-C_{j}=\prod_{k=1}^{n}\left(c_{i k}-c_{j k}\right)^{2},
$$

where $n$ is the number of genes, $c_{i k}$ is the $k$ th gene of $x_{i}$, and $c_{j k}$ is the $k$ th gene of $x_{j}$.

Therefore, according to the weight and slope of the previous moment and the current moment, the minimum value of the curved parabola is calculated, and the optimal adjustment value at the current moment is effectively calculated:

$$
A_{i j}=\sqrt{\mu\left(\frac{l_{i j}-v_{i j}}{\gamma}\right)^{2}},
$$

where $A_{i j}$ is the adjustment of the connection weight of neuron $i$ and neuron $j, l_{i j}$ and $v_{i j}$ are the partial derivative of the error function to the connection weight at the current time and the previous time, $\mu$ is the learning speed, and $\gamma$ is the proportional coefficient.

According to the combination of all elements in the structural mechanics method, the relevant parameters of the entire finite element system are calculated, for example, the equilibrium equation of any node is changed to the node displacement:

$$
V_{i}=\sum_{i=1}^{n} \frac{\alpha f_{i}-\beta f_{i}}{\rho},
$$

where $V_{i}$ is the element of the unit stiffness matrix, $f_{i}$ is the total change in fitness during this cyclic movement, $\alpha$ is the persistence of pheromone strength, $\beta$ is the attenuation of pheromone strength, and $\rho$ is the searched sliding surface safety factor.

\subsection{Artificial Intelligence-Based Calculation Model for Mine Rock Slope Stability}

2.3.1. Stability Factor Analysis. Under the condition of limited data sets, the premise of the effectiveness of artificial intelligence methods for slope stability analysis and plan optimization is to accurately extract the characteristics of the research object. At present, it is more dependent on experience and limited data analysis, such as geometric features, and material characteristics can only be assumed to be standard geometric shapes and single materials, which greatly reduce the actual number of characteristics and even loses key characteristics. Therefore, it is necessary to study new methods to obtain a large number of data sets faster and more effectively to support data-driven stability intelligent prediction and program optimization. For example, in mine slope analysis, it is often impossible to obtain a large amount of historical data. In addition, the acquisition cost of realtime monitoring data is relatively high, and the numerical model method is not restricted by natural conditions, and a large number of virtual experiments can be performed on the computer. The structural behavior is simulated under various working environments and constitutive conditions, and then a large amount of effective data reflecting the internal laws of the slope structure is generated. A relatively complete simulation and classification of the instability behavior mode has been carried out [9]. In the early stage of mining, when the monitoring data is insufficient, a virtual model of mining is constructed, and a large number of parameterized calculations are performed with the mining progress as the initial condition of the model, and virtual sensors are set at key nodes to read the calculation results to form multiple data samples. The analytical framework of stability rating of mine rock slope based on artificial intelligence is shown in Figure 1.

The genetic neural network is based on a large number of training samples. In the actual learning process, the evolutionary population can be set by the genetic algorithm, and the wrong data can be eliminated, but the accuracy of the entire model mainly depends on the accuracy of the training sample data. If the number of samples is small, the accuracy 


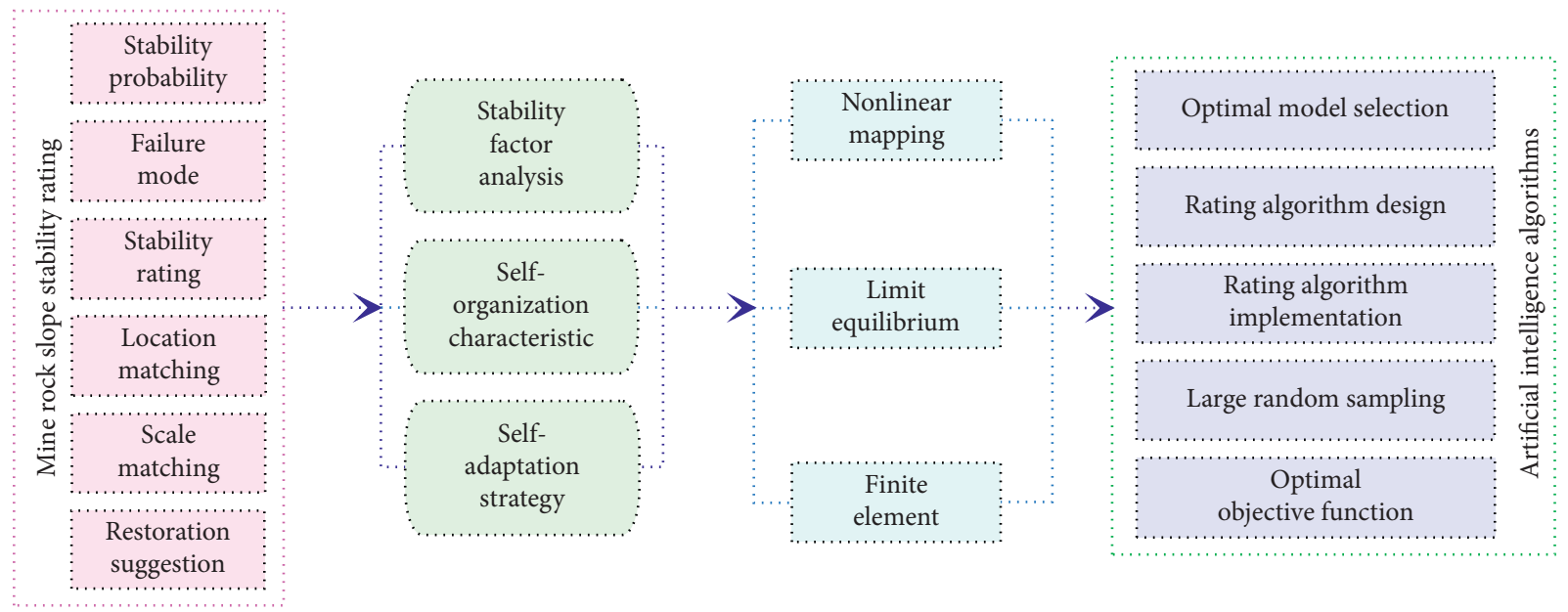

FIGURE 1: Analytical framework of stability rating of mine rock slope based on artificial intelligence.

of model prediction will be greatly affected. In a layered network, neurons are located in each layer, and only neurons in the same layer can communicate; layered networks are divided into single layer and multilayers. Compared with single-layer networks with a single function, multilayer networks have more powerful functions, and correspondingly, the calculation efficiency of the algorithm is greatly reduced. In general, genetic algorithms cannot directly deal with parameters in the space and must follow certain rules to transfer the problem to the search space that the algorithm can solve, and the process of converting the problem space into the space that the genetic algorithm can solve is the encoding. Binary coding usually needs to give the accuracy of the solution first and then determine the string length to obtain higher accuracy before algorithm operation, and the string length is usually increased. Compared with a single intelligent algorithm, combining the advantages of multiple algorithms and optimizing the compound algorithm can significantly improve the generalization performance of the model and improve its calculation accuracy.

When using the multilayer forward network for classification or function approximation, preprocessing is needed, and one of the important contents is the compression of variable dimension. When the given sample size is limited, in order to ensure that the learned network has a good generalization ability, the dimensions of the variables need to be carefully selected. Another content of the preprocessing is to normalize the input variables. Since each variable represents a different physical quantity, their value ranges may vary greatly, and it is best to normalize them to the same range of values. In the feedback neural network, the input data determines the initial state of the feedback system, and then, the system gradually converges to the equilibrium state after a series of state transitions; this equilibrium state is the calculated output result of the feedback neural network, so the stability is one of the most important problems of feedback neural networks. In the design, the factors that have a significant impact on the target problem are considered as much as possible so that the neural network will obtain better results when approximating the target problem. For a specific problem, if there are fewer hidden layer neurons to choose, the given approximation accuracy may not be obtained, and more neurons may cause learning problems. The determination of the number of input nodes is mainly based on the influencing factors of the target problem, and the number of neurons in all hidden layers is determined by the neural network design [10].

2.3.2. Optimal Model Parameter Selection. As far as the stability of the slope is concerned, the rock and soil of the slope exist objectively, and it is not vague. However, because people cannot fully grasp its laws and properties to a certain extent, they cannot be completely distinguished, which can be described qualitatively or semiquantitatively, so the understanding of the slope can only be vague. When dealing with the problem of slope stability, fuzzy uncertainty should be considered, and fuzzy mathematics' methods should be used for comprehensive identification and judgment, in order to obtain more practical results. However, only using fuzzy mathematics to analyze the stability of slopes has certain limitations, which are necessary to combine fuzzy mathematics with other mathematical methods to make up for their respective limitations and obtain better evaluation results (Figure 2). Topography is the external performance of slope stability, and the rock mass is the core of slope stability, and it is a comprehensive reflection of lithology, geological structure, and geological action of external forces. The mine rock slope's height, friction angle, slope angle, and cohesion contribute generally the same shares in the stability rating of training and test data of mine rock slope based on artificial intelligence, and sine of the rectangles, circles, diamonds, and triangles in each of the probability range is nearly evenly distributed. In order to obtain more accurate prediction results, it is necessary to study the factors that affect the stability of the slope so that the input layer contains as many influencing factors as possible, and the network structure should not be too complicated. In theory, the more factors considered, the better the description of the stable state of the slope, but, at the same time, the more complex the nonlinear relationship used to express the stability of the slope. 


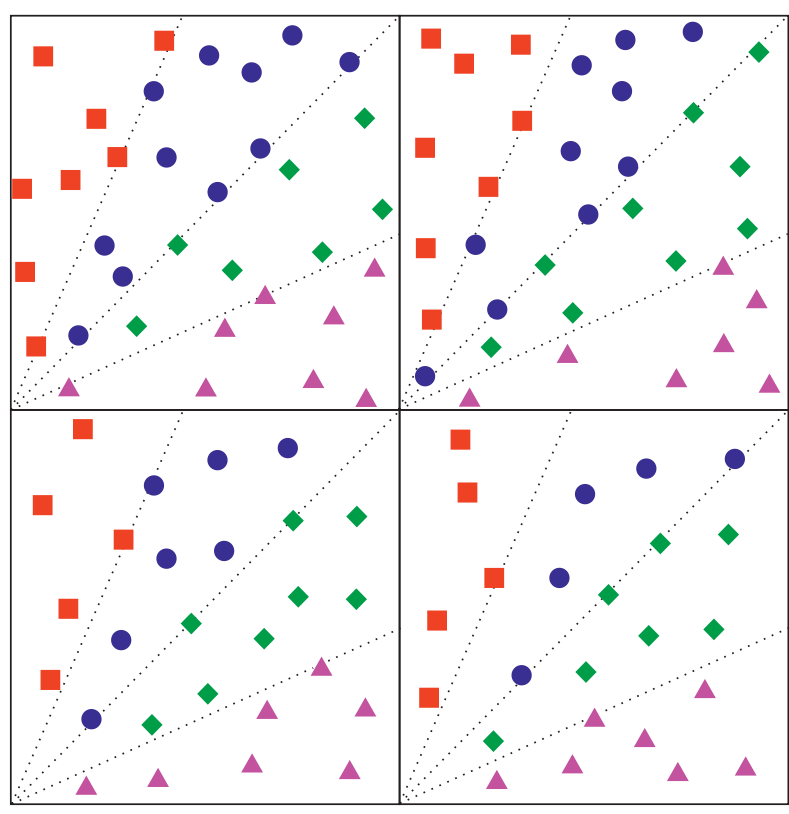

(a)

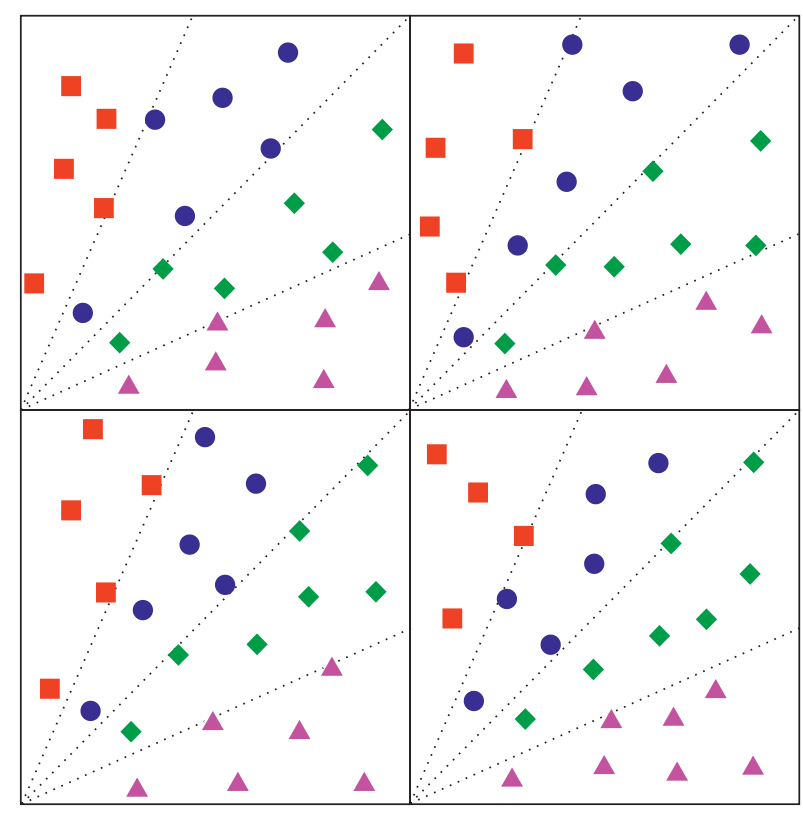

(b)

Figure 2: Rating results of training data (a) and test data (b) in the optimal model parameter selection. Note: rectangle: height; circle: friction angle; diamond: slope angle; triangle: cohesion; the three dotted lines are probability dividing lines with $25 \%, 50 \%$, and $75 \%$ probability clockwise in each subrectangle.

If the selected search area and the number of searches are reasonable enough, the pure random search method can accurately find the most dangerous sliding surface, but a large number of searches not only reduce the search efficiency but are also closely related to the operator's experience. For this reason, the random method and the optimization method can be combined to get better results, and the random search method solves the local problem better. However, according to related studies, when the section layered line and the slope surface line are both relatively complex segmented straight lines, this method cannot be completed in one search, but the final goal needs to be divided and achieved by searching multiple locations. With the development of artificial intelligence methods, some shortcomings in the limit equilibrium method have been better improved and compensated, which not only has the characteristics of the efficient random search but also can optimize multivariable, complex, and nonlinear problems and can optimize the search in the global scope [11]. In the control system, feedback refers to the process of reflecting the output to the input in a certain way and integrating it with the input according to a certain rule to determine the next decision. In the process of heterogeneous rock-slope stability analysis, the safety factor calculated by ant colony and genetic algorithm is smaller than other methods, which proves that this method can better search for the most dangerous sliding surface.

The slope engineering established in the complex geological body has extremely complex and changeable characteristics and, at the same time, has strong concealment. Therefore, in actual projects, it is best to use multiple analysis methods for comprehensive analysis at the same time according to the specific characteristics and purpose of the slope project and strive to obtain a more objective, reliable, and reasonable evaluation result. When acquiring knowledge, it only requires experts to provide examples of problems and corresponding solutions [12]. The samples are learned through specific neural network learning rules and methods, and the weights and deviations are continuously corrected by the internal adaptive algorithm of the network until the problem is reached. The required learning accuracy is to distribute the heuristic knowledge and experience of the expert to solve the problem to the parameters and connection weights between the nodes of the network. In each generation, individuals are selected according to their fitness in the problem domain, and combined crossover and mutation are performed with the help of genetic operators of natural genetics to produce a population representing a new solution set. In this way of accelerating the cycle, the change interval of the outstanding individual will gradually adjust and shrink, and the distance from the best point will be getting closer and closer, until the difference of the objective function value of the outstanding individual is less than a certain set value or the algorithm runs to reach the predetermined acceleration cycle number of times to end the operation of the entire algorithm.

\subsection{Artificial Intelligence-Based Rating Algorithm for Mine Rock Slope Stability}

2.4.1. Rating Algorithm Design. In the forward-propagation process, the input information is processed layer by layer from the input layer through the hidden layer and then passed to the output layer. If the output layer cannot get the 
expected output result, the back-propagation process is carried out, and the error signal is returned along the original connection path, and the weight and threshold of each layer of neurons are modified to make the error signal smaller. A good selection strategy must ensure that the algorithm has a faster convergence rate, and it must prevent the occurrence of premature phenomena. It not only must ensure that chromosomes with higher fitness values have a greater survival probability, but also ensure the diversity of the population. In the field of slope stability analysis, different slope projects often exist in different engineering geological environments, and different slope stability analysis methods have their own characteristics and have certain applicable conditions; the core content is the mechanical model (Figure 3). The study of mathematical models and calculation methods is the study of slope stability analysis methods. In the case of simple shape, it is assumed that the slope is destroyed along a flat or circular sliding surface. When the conditions are not single, a more complex shape of the sliding surface is used to form the corresponding analysis method. The iterative particle $x_{i} /\left(x_{i}+y_{i}\right)$ is a key step for further calculations of weight vector $\left(Q_{i j}-x_{i j}\right) / e_{i j}^{\prime \prime}$ and optimal position $p_{i} /\left(a_{i}-b_{i}\right)$, in which the analyzing conditions for node scale $1 /(n-1)$ must be satisfied first. This method is different in the degree of satisfying the equilibrium conditions, and some common analysis methods also violate the conditions of static equilibrium, which is an important factor in evaluating the accuracy of any method [13].

One of the main uncertainties in slope engineering is fuzzy uncertainty, which is mainly divided into the fuzziness of the factors affecting the stability of the slope and the uncertainty of the judgment standard of slope failure. Usually, in traditional reliability analysis, the soil parameters that affect the stability of the slope are treated as simple random variables, and sampling tests are performed at specific points. Correction to determine the soil parameters is to analyze the random uncertainty of the slope, and another major uncertainty in slope engineering is random uncertainty, resulting in a point randomness of the soil parameters. It is assumed that the mine follows the stationary hypothesis or the quasi-stationary hypothesis, that is, the parameter mean and variance have nothing to do with the location of the mine, and the autocorrelation function is only related to the relative distance between two points in space and has nothing to do with the absolute position coordinates of the two points [14]. Due to the limited on-site measured data in actual engineering, the theoretical autocorrelation function is generally used to describe the spatial autocorrelation of soil parameters. It can be seen that the cohesive force and the internal friction angle have a greater impact on the reliability of the slope, and the reliability is more sensitive to the change of the cohesive force. When only considering their variability, the failure probability of the slope is greatly increased, the reliability index is reduced, and the reliability calculation results are too conservative. Therefore, the slope reliability analysis should not be ignored, and the ambiguity of slope parameters and the slope parameters are fuzzy.
The traditional classification methods consider the above factors from their own perspectives and with different parameters, but fail to fully consider the possible failure modes of the rock mass. On the contrary, the main discontinuities associated with the slope failure mode should be fully considered. Because most rock slopes fail along the exposed geological discontinuities, the influence of discontinuities on the slope stability can be evaluated by the principle of superiority, and these classification methods can be used as a tool to describe the quality of the rock mass. When a highlevel goal is decomposed to a low-level goal, its synthesis needs to assign weights to the importance of each subgoal because the goals have different attributes and then weight the high-level goals to synthesize them. In view of the characteristics of each group of rock masses, different grading systems are used to maximize their strengths and circumvent their weaknesses. The corresponding grading system is selected as the basic tool, supplemented by comparison with other grading systems, and the expert system's combination of reasoning and calculation technology is used in the system. The rock-slope stability rating system can be used to estimate the stability of the slope, give the recommended value of the overall slope angle, determine the possible failure mode of the slope, and then propose the possible slope reinforcement method, especially suitable for the preliminary design and scheme design of the open-pit mine, as the initial work and reference conclusion of slope stability analysis.

2.4.2. Rating Algorithm Implementation. In the case of limited samples, the two-dimensional space is changed into a high-dimensional space by the nonlinear change of the inner product function, and the linear relationship between the input and output values are explored through calculation in the high-dimensional space. The algorithm of the support vector machine is to find a type of hyperplane, and it also needs to make the distance between the test data points that are relatively close to it the farthest, that is to say, the best segmentation of the hyperplane can maximize the limit of the test data points [15]. Researchers proposed the use of random search algorithms to solve the problems of support vector machines and parameter optimization. Among them, the particle swarm algorithm, genetic algorithm, ant colony algorithm, etc., are more commonly used. In many cases, the best segmentation plane sought under the condition of linear separability cannot meet the requirements in the actual use process because there may not be such a plane that can accurately divide all data points (Figure 4). If most data points are accurately under the premise of classification, there will still be a small number of data points that may be misclassified, which is called an approximately linearly separable problem. If data points that are relatively far apart can also bring significant effects on the value of the kernel function, a global kernel function such as a polynomial can be used. On the contrary, if you do not want data points that are far apart to have a significant effect on their values, choose a local kernel function such as radial basis. 


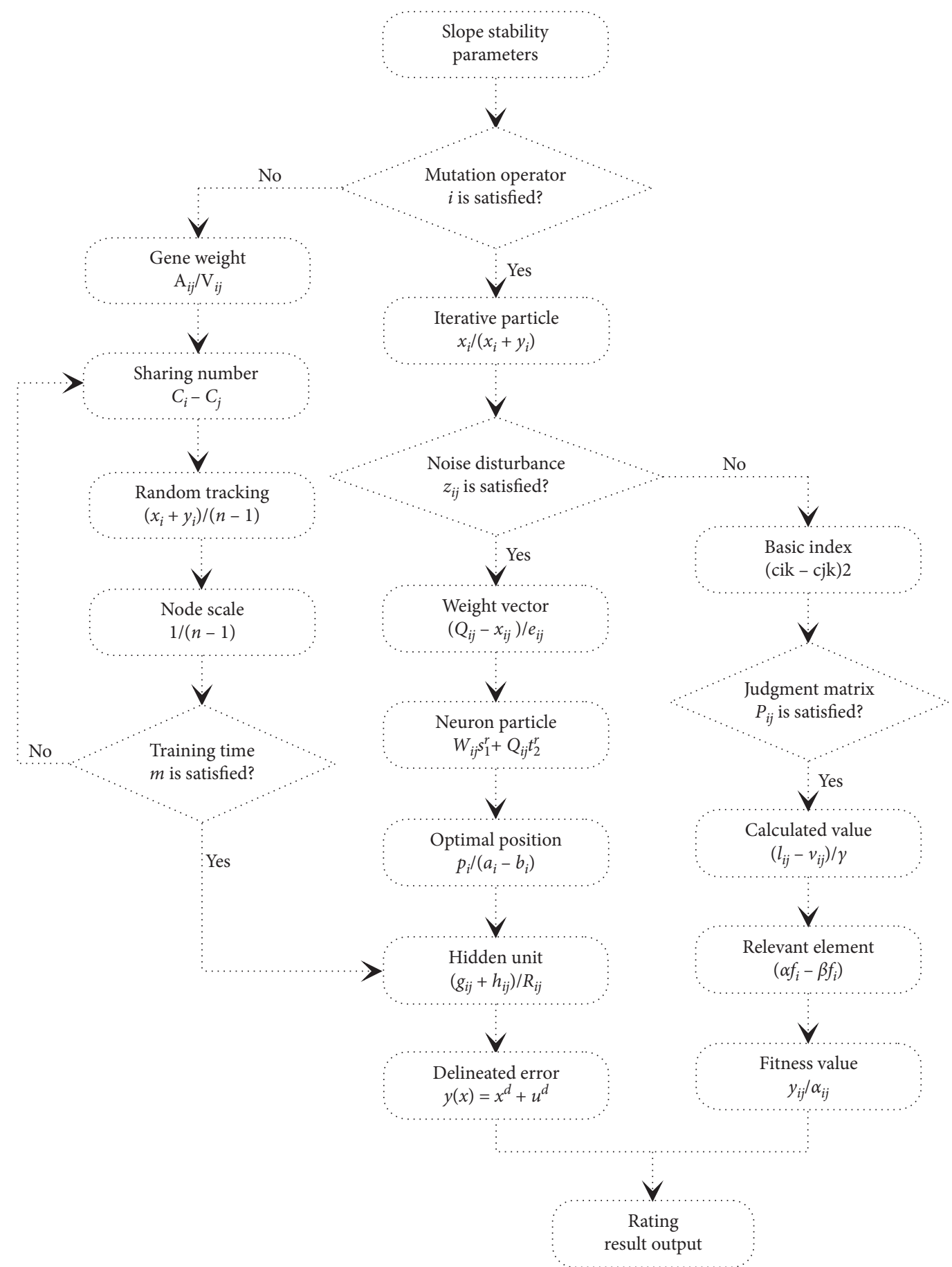

FIgURE 3: Flowchart of the artificial intelligence-based rating algorithm for mine rock slope stability.

The main purpose of slope stability analysis is to calculate the critical slip surface of the slope and the corresponding minimum safety factor with suitable mathematical programming method under given constraints, so as to provide a theoretical basis for engineering design and slope reinforcement. The limit analysis method can theoretically give the approximate range of the true solution, but the various assumptions put forward in the process of solving the upper and lower limit solutions make the upper and lower limit solutions differ from the real solution. In general, the range given is relatively large, so it is of little significance to guide engineering practice. Assuming that the properties of the material remain unchanged during each level of loading, the stress, strain, and displacement increments of the structure are obtained through linear finite element analysis. However, the physical and mechanical properties of the material change between different levels of 


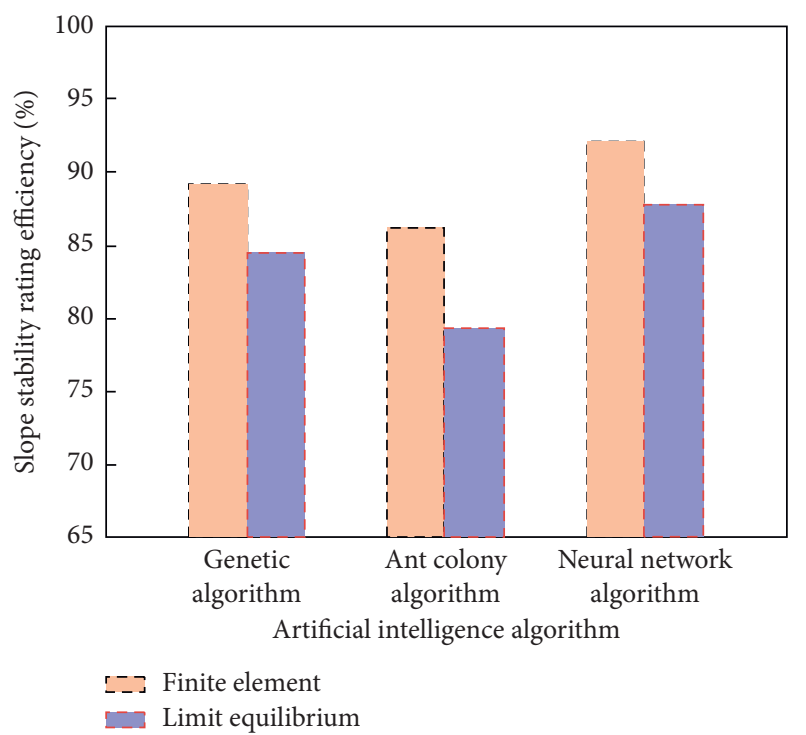

(a)

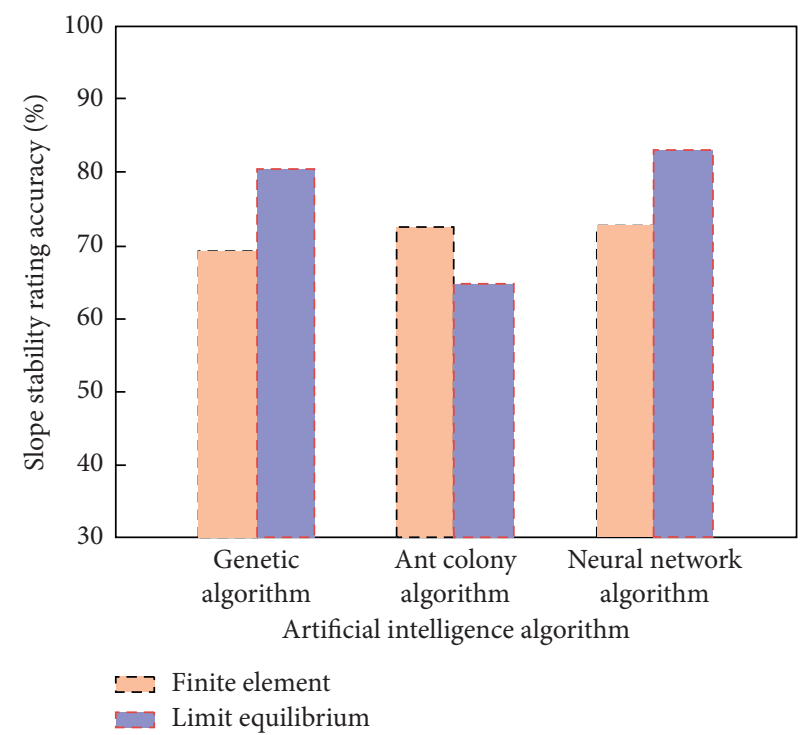

(b)

FIGURE 4: Slope stability rating efficiency (a) and accuracy (b) of three typical artificial intelligence algorithms with finite element and limit equilibrium method.

load increments, and the changing stiffness matrix reflects the nonlinear stress-strain relationship of the material. The slip surface stress analysis method not only overcomes the shortcomings of the traditional limit equilibrium method regarding the soil as a rigid body and the assumption of interstrip force distribution but also uses the finite element method to fully consider the true stress-strain relationship of the soil. The construction process and the complexity satisfy the strict mechanical equilibrium conditions and can give the advantages of soil stress and deformation information [16].

Judging from the current research, most of the slope engineering expert systems adopt manual methods to obtain expert experience knowledge, which shows that its knowledge acquisition requires multiple and repeated work, and close cooperation between slope engineers and knowledge engineers is required division of labor and science. This kind of knowledge acquisition method is effective for those expert experiences that are easy to sum up, but is powerless for those implicit parts that are not easy to express with rules. In fact, the uncertain thinking adopted by experts in solving complex slope engineering problems is multifaceted, not only vague but also random, and the slope data are also incomplete, uncertain, and contains noise. The integration of traditional and nontraditional representation methods, the display and representation of deep knowledge, and the representation of common sense knowledge will be the main tasks faced by the slope engineering expert system in the future [17]. Therefore, a comprehensive study of uncertain reasoning models from the aspects of fuzziness, randomness, incomplete information, and uncertainty will have a broader significance. The integration of various reasoning methods and technologies, the combination of symbols and calculations, the combination of qualitative reasoning and quantitative calculations, heuristic reasoning, and model-based research will also be important topics.

\subsection{Case Analysis}

2.5.1. Mine Slope Overview. The mine slope in the selected area in this paper extends along the NW-SE direction, with a slope of $960 \mathrm{~m}$. The highest elevation of the slope is $1140 \mathrm{~m}$; the lowest elevation is $980 \mathrm{~m}$; the height of the slope is $340 \mathrm{~m}$; the width is $490 \mathrm{~m}$; the aspect is $217^{\circ}$; the slope angle is $14^{\circ}$. According to the change of the slope angle parameters of the step slope, the overall slope is divided into 3 combined step slopes. Combined step slope I: $900-1050 \mathrm{~m}, 26 \mathrm{~m}$ step height, $21 \mathrm{~m}$ platform width, $38^{\circ}$ step slope angle, and $11^{\circ}$ mining slope angle; the combined step slope II: $1010-1190 \mathrm{~m}, 39 \mathrm{~m}$ step height, $35 \mathrm{~m}$ platform width, $42^{\circ}$ step slope angle, and $19^{\circ}$ mining slope angle; the combined slope III: $1120-1290 \mathrm{~m}, 25 \mathrm{~m}$ step height, $22 \mathrm{~m}$ platform width, $53^{\circ}$ step slope angle, and $20^{\circ}$ mining slope angle. Rhyolite, tuff, and dacite are exposed in the slope rock mass from bottom to top, in which the dacite develops a complete weathering profile with freshly unweathered, lightly weathered, moderately weathered, strongly weathered, and fully weathered. There are 4 faults in the slope area, and the tuff is distributed in layers, forming a weak interlayer of the slope rock mass. There are generally 3 groups of joints in the slope area, and a group of weathered joints are also developed in the strongly weathered dacite. Figure 5 shows the study case mine rock slope with its constituent elements and calculation model.

This simple and vivid local safety factor method that considers the progressive destruction of rock slopes, combined with the newly generated harmony search algorithm and the nonuniform mutation operator in the genetic 


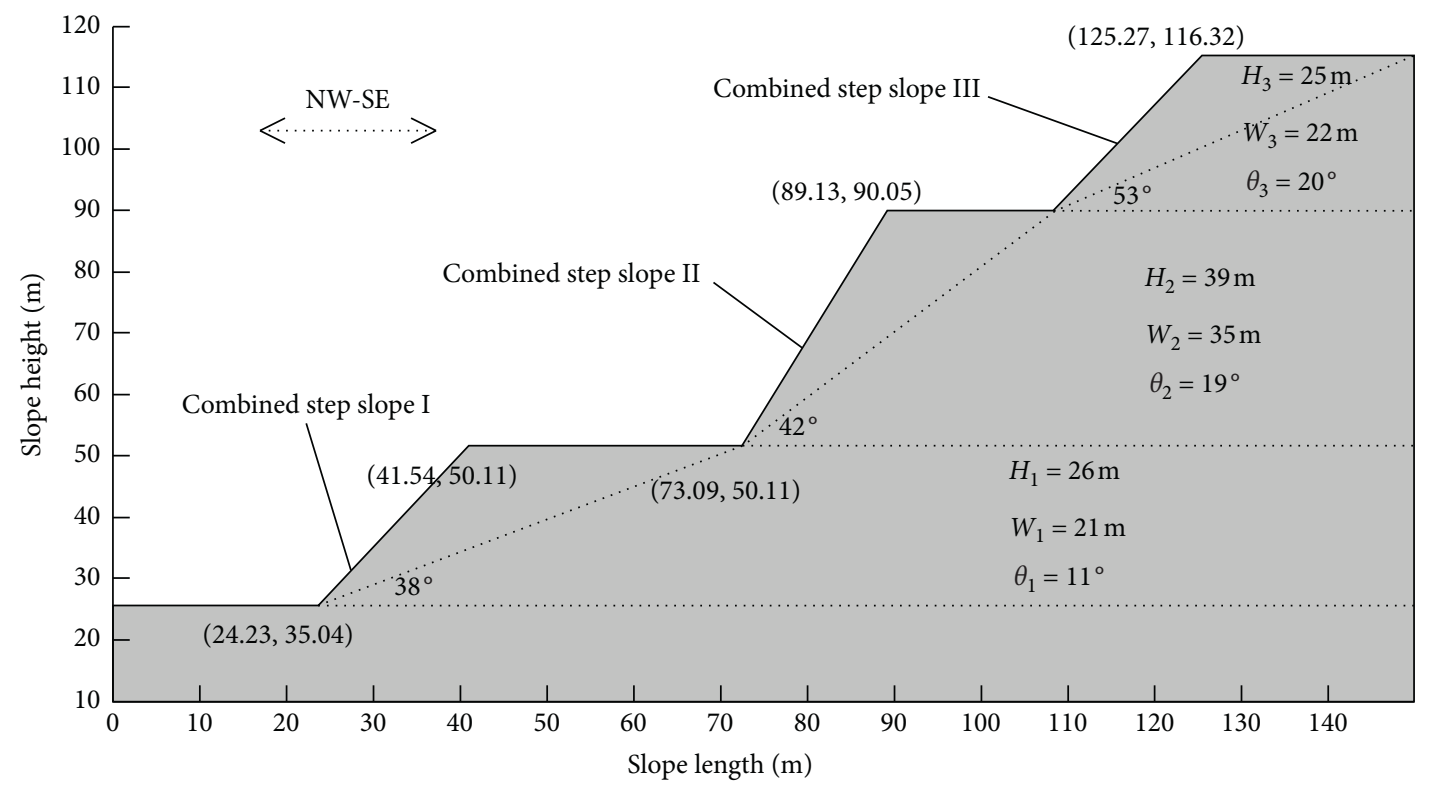

Figure 5: Study case mine rock slope with constituent elements and calculation model.

algorithm, proposes a harmony search algorithm that simulates the maximum, medium, and minimum optimization process. The stability analysis of simple rock slopes, heterogeneous rock slopes, and unstable rock slopes are carried out, and compared with the existing results, it is found that the method in this paper can be used as a new attempt to calculate the stability of rock slopes. Any global optimization method must coordinate the development and exploration of the solution space. If the development ability of the algorithm is overemphasized, the algorithm is easy to fall into the local optimum. The search capability of the twosided mapping shrinkage operator is higher than that of the one-sided mapping shrinkage operator. It uses the largest redundant point, the vertex corresponding to the largest pseudogradient, and the largest information entropy to search the solution space, which is better than the basic compound shape method [18]. Although the new optimization algorithm has strong global search ability, its calculation parameters are often difficult to determine, which makes the algorithm easy to fall into local optimal values. The basic harmony algorithm does not fully utilize the information in the harmony library when searching. A genetic algorithm that introduces harmony strategies and an improved harmony algorithm is proposed, and the comparison results show that the proposed algorithm can search for a smaller safety factor.

2.6. Result Analysis. The artificial intelligence-based mine rock slope stability rating system is actually a large-scale continuous time-dynamic system with a high degree of nonlinearity, which has the common characteristics of general nonlinear dynamic systems, such as attractiveness, nonequilibrium, irreversibility, high dimensionality, extensive connectivity, and adaptability, but also has distinct characteristics, such as fault tolerance, large-scale parallel distributed processing, high robustness, and learning association ability, which is powerless for those implicit parts that are not easy to express with rules. Because fuzzy control technology has an accurate mathematical model that does not depend on the controlled object, it is simple in design, easy to apply, strong in anti-interference ability, and fast in response. It is easy to control and master and has strong robustness to the changes of system parameters, which is mostly used to control uncertain systems (Figure 6). It is precisely because of the variability in space, time, and engineering properties of the rock and soil that constitute the slope; the search for the critical slip surface has evolved into a complex, nonlinear, and nonconvex programming problem. Since the determined safety factor value cannot truly characterize the safety of the slope, it is necessary to consider the randomness of slope analysis and design parameters and apply random theory to evaluate the reliability of slope engineering [19].

According to the basic stress equation and related yield conditions of the plane strain problem, a first-order quasilinear hyperbolic partial differential equation group can be obtained, and then, two characteristic line differential equations can be decomposed, and this characteristic line is the slip line of the soil. This differential equation needs to be solved by integral, and only a few cases have analytical solutions, such as ignoring the soil's own weight, and more general cases require numerical methods. The vertical strip method and the oblique strip-mode energy method have their own characteristics, and simple discrete methods are used to solve the corresponding safety factors [20]. The vertical striping method was developed earlier, and the concept is simpler and more straightforward, but it is not as strict as the diagonal striping method from the theoretical basis. When the population size is small, the greater the evolutionary algebra, and its search ability is obviously better than that of the less evolutionary generation. When the 


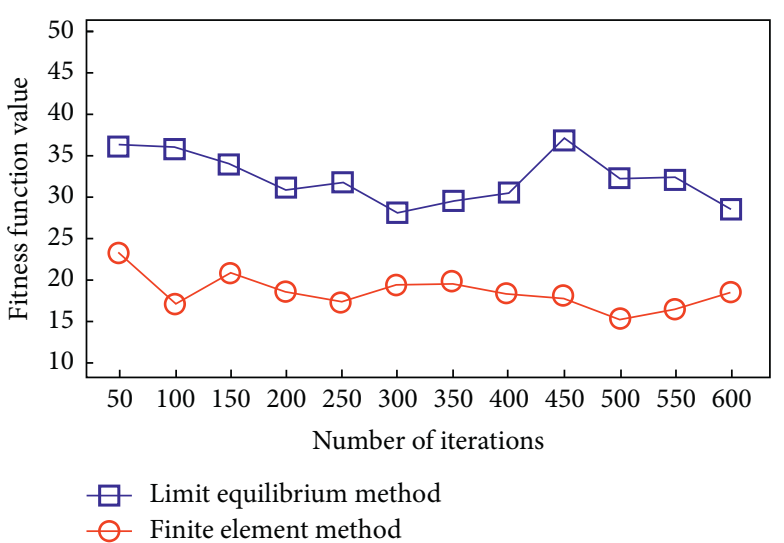

(a)

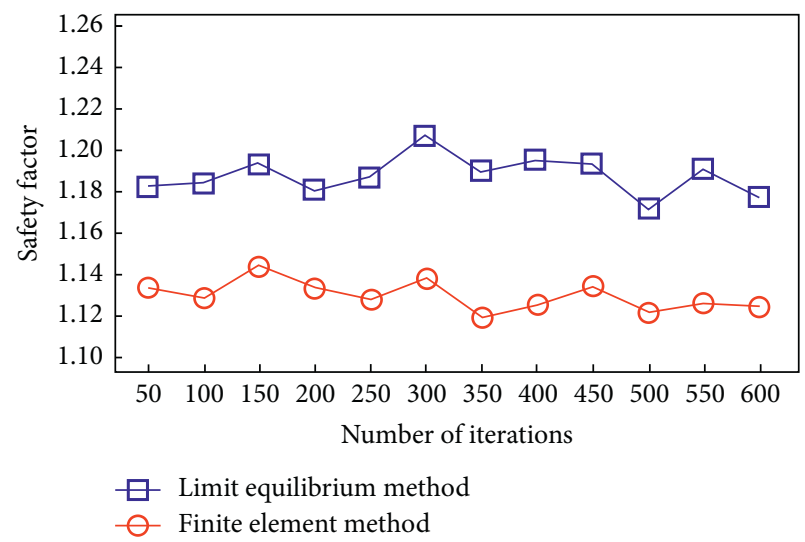

(c)

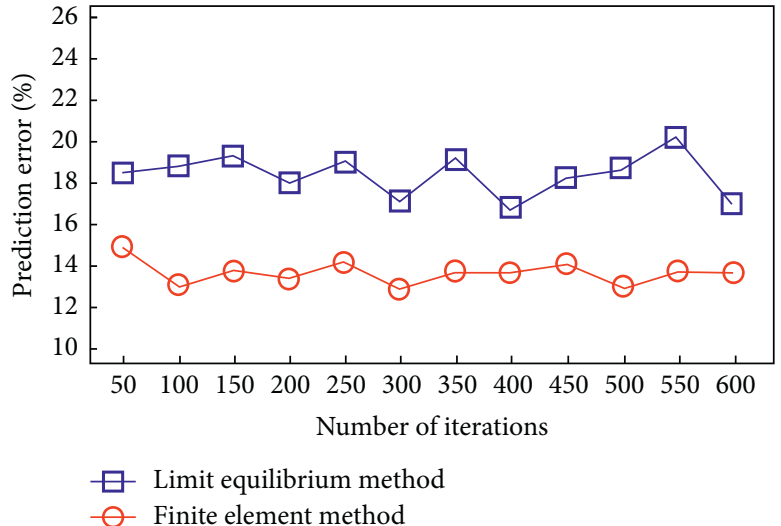

(b)

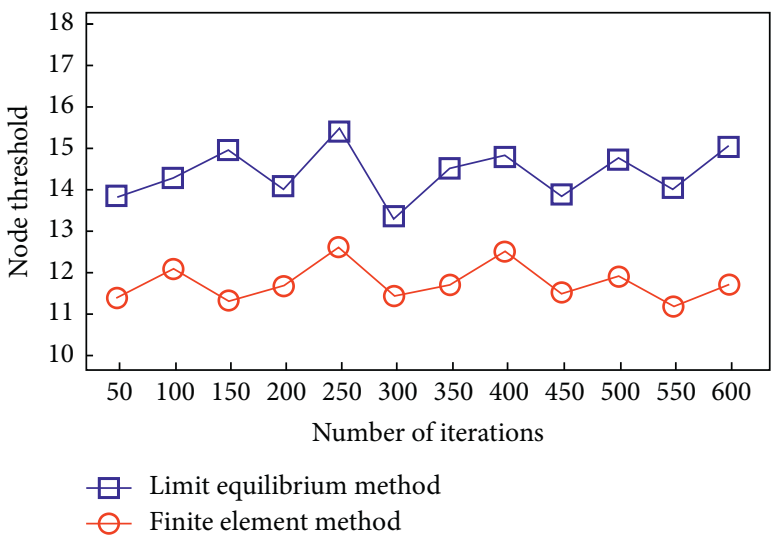

(d)

Figure 6: Fitness function value (a), prediction error (b), safety factor (c), and node threshold (d) of the limit equilibrium method and finite element method in mine rock slope stability rating.

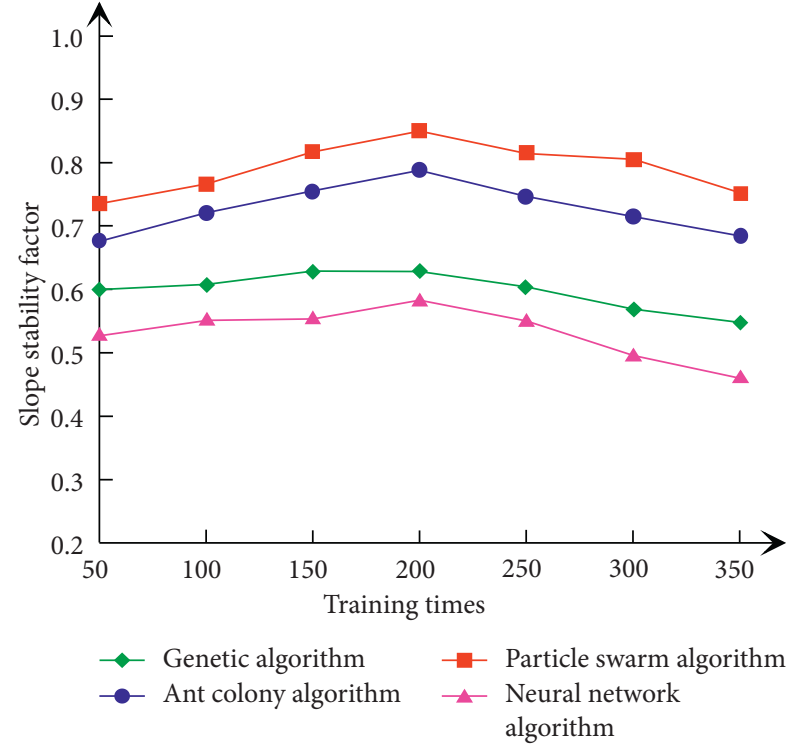

(a)

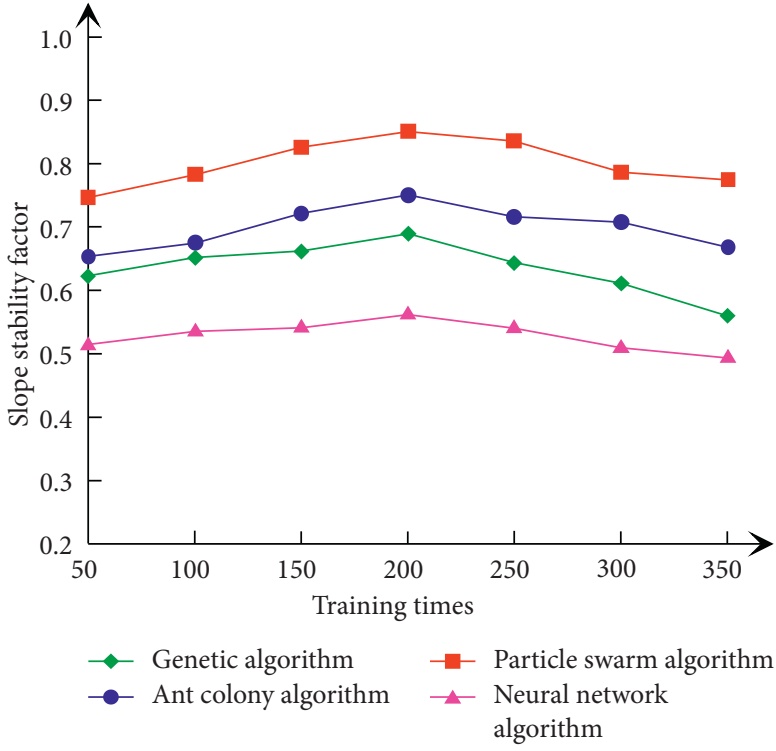

(b)

Figure 7: Slope stability factor of different artificial intelligence algorithms in the limit equilibrium method (a) and finite element method (b). 
population size reaches a certain number, the evolutionary algebra has little influence on the optimization result, and this problem is an optimization problem under multidimensional constraints (Figure 7). When the population size is large, the evolutionary algebra has less influence on the optimization results of the algorithm, and the optimization results under different evolutionary algebras are basically close. The traditional method of determining the sliding surface of a slope is by determining the coordinates of the center of the sliding surface. However, the proposal of an algorithm needs to be considered from many aspects, especially the specific problem of the algorithm and the stability shown during the search [21].

When analyzing the slope, if the considerations are not comprehensive enough, the results of the built model will be far from the actual situation. This means that it is difficult to use accurate and reliable mathematical models to reflect the highly nonlinear formation mechanism and mechanical behavior process. First, only when the scale of the training sample data is large enough to reach infinity, the empirical risk obtained according to the law of large numbers can approach the expected risk in terms of probability, which does not mean that the parameters in the two functions are of the same value. It does not mean that the empirical risk can be close to the expected risk; even if these conditions are guaranteed under the support of infinite sample data, the empirical risk obtained under the limited sample data may not be close to the expected risk. The generalized boundary is the relationship between the empirical risk and actual risk [22]. Whether the generalized boundary can be handled correctly, it is particularly important to analyze the performance of the learning machine and improve the development algorithm. For more complex problems such as slope stability, the radial basis kernel function can play its role well and has better approximation performance for nonlinear problems. Using this for sample training, the model establishes a slope safety factor prediction model, which contains a nonlinear mapping relationship between stability influencing factors and safety factors.

\section{Conclusions}

This paper constructs an artificial intelligence-based mine rock slope-stability calculation model, performs slope stability influencing factors' analysis and optimization model parameter selection, proposes a mine rock slope stability rating algorithm based on artificial intelligence, analyzes the rating algorithm design and rating algorithm implementation, and finally conducts a case analysis and its discussion. When analyzing the slope, if the considerations are not comprehensive enough, the results of the built model will be far from the actual situation. This means that it is difficult to use accurate and reliable mathematical models to reflect the highly nonlinear formation mechanism and mechanical behavior process, and the genetic neural network is based on a large number of training samples. The corresponding grading system is selected as the basic tool, supplemented by comparison with other grading systems, and the expert system's combination of reasoning and calculation technology is used in the system. In the actual learning process, the evolutionary population can be set by the genetic algorithm, and the wrong data can be eliminated, but the accuracy of the entire model mainly depends on the accuracy of the training sample data. The study results show that the artificial intelligence-based mine rock slope stability rating method can make full use of computer advantages to have larger capacity and faster calculation speed and solve to the greatest extent the randomness, timeliness, ambiguity, and other uncertain characteristics of mine rock slope stability by a large number of random sampling for finding the optimal value of the objective function. The study results of this paper provide a reference for further research studies on the stability rating of mine rock slopes based on artificial intelligence.

\section{Data Availability}

No data were used to support the findings of the study.

\section{Consent}

Informed consent was obtained from all individual participants included those in the study references.

\section{Conflicts of Interest}

The author declares that there are no conflicts of interest.

\section{References}

[1] C. Yuan and H. Moayedi, "The performance of six neuralevolutionary classification techniques combined with multilayer perception in two-layered cohesive slope stability analysis and failure recognition," Engineering with Computers, vol. 36, no. 4, pp. 1705-1714, 2020.

[2] S. Suman, S. Z. Khan, S. K. Das, and S. K. Chand, "Slope stability analysis using artificial intelligence techniques," Natural Hazards, vol. 84, no. 2, pp. 727-748, 2016.

[3] H. Fattahi, "Prediction of slope stability using adaptive neurofuzzy inference system based on clustering methods," Journal of Mining and Environment, vol. 8, no. 2, pp. 163-177, 2017.

[4] B. Gordan, D. Jahed Armaghani, M. Hajihassani, and M. Monjezi, "Prediction of seismic slope stability through combination of particle swarm optimization and neural network," Engineering with Computers, vol. 32, no. 1, pp. 85-97, 2016.

[5] T. Hemalatha, M. V. Ramesh, and V. P. Rangan, "Effective and accelerated forewarning of landslides using wireless sensor networks and machine learning," IEEE Sensors Journal, vol. 19, no. 21, pp. 9964-9975, 2019.

[6] Y. M. Ospina-Dávila and M. Orozco-Alzate, "Parsimonious design of pattern recognition systems for slope stability analysis," Earth Science Informatics, vol. 13, no. 2, pp. 523-536, 2020.

[7] F. Tian, H. Pang, X. Sun, and C. Wang, "A kind of slope stability evaluation model based on SVM-DS method," International Journal of Autonomous and Adaptive Communications Systems, vol. 8, no. 2/3, pp. 141-149, 2015.

[8] S.-H. Jiang, D.-Q. Li, Z.-J. Cao, C.-B. Zhou, and K.-K. Phoon, "Efficient system reliability analysis of slope stability in 
spatially variable soils using Monte Carlo simulation," Journal of Geotechnical and Geoenvironmental Engineering, vol. 141, no. 2, 2015.

[9] A. H. Abd and S. Utili, "Design of geosynthetic-reinforced slopes in cohesive backfills," Geotextiles and Geomembranes, vol. 45, no. 6, pp. 627-641, 2017.

[10] J. Ji, C. Zhang, Y. Gao, and J. Kodikara, "Effect of 2D spatial variability on slope reliability: a simplified FORM analysis," Geoscience Frontiers, vol. 9, no. 6, pp. 1631-1638, 2017.

[11] A. H. Gandomi, A. R. Kashani, M. Mousavi, and M. Jalalvandi, "Slope stability analysis using evolutionary optimization techniques," International Journal for Numerical and Analytical Methods in Geomechanics, vol. 41, no. 2, pp. 251-264, 2017.

[12] X. Xue, Y. Li, X. Yang, X. Chen, and J. Xiang, "Prediction of slope stability based on GA-BP hybrid algorithm," Neural Network World, vol. 25, no. 2, pp. 189-202, 2020.

[13] M. Rahimi, Z. Wang, A. Shafieezadeh, D. Wood, and E. J. Kubatko, "Exploring passive and active metamodelingbased reliability analysis methods for soil slopes: a new approach to active training," International Journal of Geomechanics, vol. 20, no. 3, Article ID 04020009, 2020.

[14] H. Moayedi, M. Mosallanezhad, A. S. A. Rashid, W. A. W. Jusoh, and M. A. Muazu, "A systematic review and meta-analysis of artificial neural network application in geotechnical engineering: theory and applications," Neural Computing and Applications, vol. 32, no. 2, pp. 495-518, 2020.

[15] H. Wu, Y. Guo, L. Xiong, W. Liu, G. Li, and X. Zhou, "Optical fiber-based sensing, measuring, and implementation methods for slope deformation monitoring: a review," IEEE Sensors Journal, vol. 19, no. 8, pp. 2786-2800, 2019.

[16] A. P. Dyson and A. Tolooiyan, "Optimisation of strength reduction finite element method codes for slope stability analysis," Innovative Infrastructure Solutions, vol. 3, no. 1, Article ID 38, 12 pages, 2018.

[17] M. A. Hossain and A. Islam, "Numerical analysis of the effects of soil nail on slope stability," International Journal of Computer Applications, vol. 141, no. 8, pp. 12-15, 2016.

[18] C. Qi and X. Tang, "A hybrid ensemble method for improved prediction of slope stability," International Journal for $\mathrm{Nu}$ merical and Analytical Methods in Geomechanics, vol. 42, no. 15, pp. 1823-1839, 2018.

[19] D. K. Yadav, G. Karthik, S. Jayanthu, and S. K. Das, "Design of real-time slope monitoring system using time-domain reflectometry with wireless sensor network," IEEE Sensors Letters, vol. 3, no. 2, pp. 1-4, 2019.

[20] G. J. Hearn and A. B. Hart, "Landslide susceptibility mapping: a practitioner's view," Bulletin of Engineering Geology and the Environment, vol. 78, no. 8, pp. 5811-5826, 2019.

[21] B. Jellali and W. Frikha, "Constrained particle swarm optimization algorithm applied to slope stability," International Journal of Geomechanics, vol. 17, no. 12, Article ID 06017022, 2017.

[22] V. Villani, G. Rianna, P. Mercogliano, A. L. Zollo, and P. Schiano, "Statistical approaches versus weather generator to downscale $\mathrm{rcm}$ outputs to point scale: a comparison of performances," Journal of Urban and Environmental Engineering, vol. 8, no. 2, pp. 142-154, 2015. 